\section{EM training}

To the editor: The useful survey presented by Bhimani and colleagues ${ }^{1}$ may (or may not) have been confounded by question 6 regarding $\mathrm{CCFP}(\mathrm{EM})$ training. It may not distinguish those who have completed a third year of emergency medicine education from physicians who have achieved the CCFP(EM) designation by the practice-eligible route. This, of course, depends on the instructions given to the survey participants.

\section{Seamus Donaghy}

Grimsby, Ont.

\section{Reference}

1. Bhimani M, Dickie G, McLeod S, et al. Emergency medicine training demographics of physicians working in rural and regional southwestern Ontario emergency departments. CJEM 2007;9: 449-52.

\section{[The authors respond]}

To the editor: As noted, our survey did not distinguish between CCFP(EM) certified physicians who had residency training and those who had practice eligible training. As the College of Family Physicians of Canada treats holders of this designation equally, we would assume that they clinically represent similar practice skills. The CCFP(EM) designation entails passing a rigorous examination, and practice eligible candidates also need to demonstrate many hours of defined emergency medicine work. For the purpose of our survey, any holder of the CCFP(EM) designation would be assumed to have an additional emergency medicine skill set.

\section{Munsif Bhimani, MSc, MD}

London Health Sciences Centre and Division of Emergency Medicine
Schulich School of Medicine and

Dentistry

University of Western Ontario

London, Ont.

Gordon Dickie, MB, ChB

Schulich School of Medicine and

Dentistry

University of Western Ontario

London, Ont.

Shelley McLeod, MSc, BSc

London Health Sciences Centre

London, Ont.

Daniel Kim MD, BSc

University of Toronto

Toronto, Ont.

\section{Procedural sedation}

To the editor: The article by Zed and colleagues ${ }^{1}$ raises some interesting questions. Could it be that contrary to current belief, propofol does have analgesic/amnesic properties? It would be helpful to know which patients (and their procedures) were given fentanyl. Which patients (and procedures) experienced recall? I suggest that $6.2 \%$ recall of a painful procedure is unacceptable. The addition of $1 \mathrm{mg}$ to $2 \mathrm{mg}$ midazolam may be a small price to pay for total absence of recall. Since 1 of the goals of procedural sedation in the emergency department is to have the patient "street ready" in the shortest time possible, is it now time to consider the use of alfentanil or, better still, remifentanil, given the brief duration of pain inflicted by the majority of our emergency department interventions?

\section{Seamus Donaghy}

Grimsby Ont.

\section{Reference}

1. Zed PJ, Abu-Laban RB, Chan WWY, et al. Efficacy, safety and patient satisfaction of propofol for procedural seda- tion and analgesia in the emergency department: a prospective study. CJEM 2007;9:421-7.

\section{[The authors respond]}

To the editor: We thank Dr. Donaghy for his letter regarding our paper. ${ }^{1}$ It is clear that propofol has very effective amnestic properties, as demonstrated by the lack of procedural recall in $93.8 \%$ of patients in our study. Of the 113 patients in our study, fentanyl, the use of which was left to the discretion of the emergency physician, was administered in $19(16.8 \%)$. These 19 patients had a mean age of 40.3 (SD 15.4) years and $73.7 \%$ were male. The procedures included 12 orthopedic manipulations, 3 abscess incision and drainages, 2 chest tube insertions, 1 foreign body removal and 1 incarcerated hernia reduction. Recall was absent in 17 patients (89.4\%).

Overall, recall was reported in 7 patients $(6.2 \%)$; 3 reported a pain score of 0 on a 10 -point visual analog scale, and the remaining 4 patients reported pain scores of 2, 4, 4 and 5, respectively. Among the 4 patients that reported recall and pain, 2 (50\%) (both shoulder dislocations) were given $100 \mathrm{mcg}$ of fentanyl prior to the administration of propofol.

Dr. Donaghy raises intriguing questions regarding the appropriate use of analgesics in the setting of procedural sedation and analgesia (PSA). The current literature on this aspect of propofol suggests further study is required. ${ }^{2}$ Our study does little to clarify the situation, since only 4 patients $(3.5 \%)$ reported recall and pain, and one-half of them received fentanyl, underscoring the difficulty in predicting the analgesic requirements in this patient population. A recent study published in abstract form by Miner and colleagues ${ }^{3}$ evaluated the 